\title{
A PRODUÇÃO INTERNACIONAL EM RBV: CARACTERÍSTICAS GERAIS E TRATATIVA DOS RECURSOS NA PESQUISA EM ORGANIZAÇÕES DE MENOR PORTE
}

\section{RESUMO}

Desde a década de 80, a Resource-based View (RBV) tem-se destacado como uma das principais correntes da estratégia. O volume e a complexidade da produção intelectual em RBV colocam àquele que se propõe transitar pela teoria o desafio de compreender suas características gerais. Sendo a teoria formatada preponderantemente a partir da análise da grande organização, acresce-se o desafio de entender a tratativa conferida aos recursos na pesquisa em organizações de menor porte. Perscrutando os acervos de alguns dos mais impactantes periódicos internacionais, a pesquisa relatada neste artigo tem por objetivo apresentar indicativos qualificadores e afigurações temáticas da produção em RBV. As análises apoiam-se em dados estatísticos e explorações textuais referentes ao conjunto considerado. Os resultados apontam para a larga prevalência de estudos quantitativos e, como objeto de pesquisa, de grandes organizações. A pesquisa em organizações de menor porte é, na sequência, analisada, e dela são extraídas as diretrizes norteadoras e as tratativas conferidas aos recursos. O artigo contribui, dessa forma, para o deslindamento da configuração da teoria, propondo, concomitantemente, sugestões para sua extensão.

Palavras-chave: Resource-based View (RBV); Produção Intelectual; Mapeamento Bibliográfico; Recursos; Organizações de Menor Porte.

INTERNATIONAL PAPERS PUBLISHED ON RESOURCE-BASED VIEW: GENERAL CHARACTERISTICS AND THE WAY RESOURCES ARE TREATED ON THE RESEARCH WITH SMALLER ORGANIZATIONS

\section{ABSTRACT}

Since the 80s, the Resource-based View (RBV) has been one of the main theories of strategic management. The volume and the complexity of RBV publications challenge the researcher to understand its general characteristics. As the theory is mainly based on the analysis of large organizations, the researcher is also challenged to understand how resources are treated on researches that deal with smaller organizations. Analysing texts published in some of the most important international academic journals, this paper aims to present indicators that show how the field has been developed. The analyses rely on statistical and textual data. The results suggest the wide prevalence of quantitative methods and the choice by large organizations as object of study. The research with smaller organizations is then analysed. The research guidelines are presented, as well as the way the resources are treated. The paper contributes thus to the comprehension of the field configuration and displays concomitantly suggestions for its extension.

Keywords: Resource-based View (RBV); Academic Publication; Bibliographic Mapping; Resources; Smaller Organizations. 
A Produção Internacional em RBV: Características Gerais e Tratativa dos Recursos na Pesquisa em Organizações de Menor Porte

\section{LA PRODUCCIÓN CIENTÍFICA INTERNACIONAL EN RBV: CARACTERÍSTICAS GENERALES Y TRATO DE LOS RECURSOS EN LA PESQUISA EN ORGANIZACIONES DE MENOR TAMAÑO}

\section{RESUMEN}

Desde la década de 80, la Resource-based View (RBV) se ha destacado como una de las principales corrientes de la estrategia. El volumen y la complejidad de la producción intelectual en RBV han desafiado la comprensión a los muchos investigadores. La teoría está formateada principalmente del análisis de gran organización, lo que plantea el desafío de la comprensión de cómo se tratan los recursos en la investigación en las organizaciones más pequeñas. Este artículo tiene como objetivo analizar los textos publicados en algunas de las revistas internacionales de mayor impacto para presentar características de la producción, especialmente en la investigación en las organizaciones de menor tamaño. Los resultados apuntan a la amplia prevalencia de estudios cuantitativos y de las grandes organizaciones como objeto de investigación. En una parte del artículo, se analiza específicamente la investigación en las organizaciones de menor tamaño. Así pues, el artículo contribuye a una mejor comprensión del campo, proponiendo concomitantemente sugerencias para su ampliación.

Palabras-clave: Resource-based View (RBV); La Producción Intelectual; Mapeo Bibliográfica; Recursos; Las Organizaciones de Menor Tamaño.

Ricardo Lebbos Favoreto ${ }^{1}$ Saulo Fabiano Amâncio-Vieira ${ }^{2}$

\footnotetext{
${ }^{1}$ Doutor em Administração pela Universidade Nove de Julho - UNINONE. Professor da Universidade Estadual do Paraná - UNESPAR. Brasil. E-mail: ricardo.favoreto@ hotmail.com

${ }^{2}$ Doutor em Administração pela Universidade Nove de Julho - UNINONE. Professor da Universidade Estadual de Londrina - UEL. Brasil. E-mail: $\underline{\text { saulo@ uel.br }}$
} 


\section{INTRODUÇÃ̃O}

A RBV tem-se destacado, desde a década de 1980, como uma das principais teorias da estratégia (Hoopes, Madsen \& Walker, 2003; Newbert, 2007; Stieglitz \& Heine, 2007). Ocupou o editorial dos mais notórios periódicos da área e influenciou sobremaneira o pensamento atual em gestão estratégica. Fundamentalmente, sugere basear em recursos a compreensão da estratégia organizacional, conforme se pode inferir do artigo que a titulou (Wernerfelt, 1984).

Debruçando-se os estudos iniciais sobre a formulação de proposições teóricas, constituintes do alicerce conceitual da teoria, a partir de dado momento, propagaram-se pesquisas empíricas, realizadas sobre o suporte de teorias variadas. Dotada de um framework encorajador do diálogo entre perspectivas distintas, a RBV cativou muitos pesquisadores, permeando-se extensamente pelo campo da estratégia (Mahoney \& Pandian, 1992). Congregou qualificativos como: "uma das mais populares e frutíferas áreas da pesquisa em estratégia" (Hoopes et al., 2003, p. 889) e "uma das mais largamente aceitas teorias da gestão estratégica" (Newbert, 2007, p. 121).

Com a sistematização da teoria, alvidrava-se a alteração do enfoque então vigente no campo da estratégia: da análise baseada nas forças da indústria (Porter, 1980), para a consideração dos efeitos específicos da firma (Spanos \& Lioukas, 2001). Contudo, a despeito das mudanças no enfoque e no nível de análise, a pesquisa em RBV, seguindo a tradição estabelecida no campo, permaneceu direcionada para o estudo da grande organização. Daí decorrem, por consequência, seus construtos e suas premissas: a formatação do seu mainstream.

No artigo de revisão sobre os dez anos que se seguiram ao artigo de 1991, Barney, Wright e Ketchen (2001) constataram que muito do foco da pesquisa em RBV concentrara-se em grandes empresas, enquanto empresas menores também enfrentam a necessidade de adquirir recursos críticos para a criação de vantagem competitiva. O empreendedorismo estratégico (Meyer, Neck \& Meeks, 2002), constituindo-se de pesquisas muitas das quais realizadas no âmbito da pequena e média empresa, pode ser visto, de certa forma, como um reflexo da demanda pela extensão das estruturas do campo da estratégia para a apreensão do fato estratégico ocorrido nas organizações de menor porte. Tal desenvolvimento alinha-se à diligência de reforçar a impressão de mais contexto na pesquisa em estratégia, superando-se a prática de se adotar como objeto a grande corporação e se tomarem como referência modelos americanos (Whittington, Pettigrew \& Thomaz, 2002).

$\mathrm{O}$ volume e a complexidade da produção em RBV conduzem o pesquisador ao desafio de compreender suas características gerais. A formatação preponderante a partir da análise da grande organização acresce o desafio da compreensão da tratativa conferida aos recursos na pesquisa em organizações de menor porte. O artigo de Acedo, Barroso e Galan (2006) talvez o mais notório estudo sobre a organização estrutural da produção em RBV - e os impactos resultantes reforçaram os potenciais benefícios advindos desse tipo de exame. A caracterização da produção intelectual em determinada teoria dá, elementarmente, a conhecer o estado da arte correspondente, possibilitando ao pesquisador um retrato das ocorrências produtivas referentes.

Procede-se, neste artigo, à revisão da produção intelectual em RBV veiculada em alguns dos mais notórios periódicos internacionais. A pesquisa conduziu-se pelo objetivo geral de extrair do conjunto analisado indicativos qualificadores da teoria $\mathrm{e}$ afigurações temáticas das tratativas conferidas aos recursos na pesquisa em organizações de menor porte. Para tanto, percorreram-se os seguintes objetivos intermediários: i) compilar os textos em RBV integrantes dos acervos dos periódicos selecionados; ii) constituir um rol de indicativos a partir dos quais se possa categorizar os textos; iii) abstrair dos textos compilados os que relatam pesquisas realizadas em organizações de menor porte; iv) identificar e categorizar as tratativas conferidas aos recursos nesses textos.

\section{RBV: PANORAMA TEÓRICO SOB A ÓTICA DE RECURSOS E ALGUNS ASPECTOS GERAIS}

O interesse havido no campo pela consideração dos recursos orienta-se especialmente pela questão do papel dos recursos na consecução de vantagens competitivas (Wernerfelt, 1984). A organização pode ser entendida como um feixe de recursos, decorrendo as resultantes ofertadas ao mercado diretamente do modo como são eles operados (Penrose, 1997).

Contêm-se os antecedentes originários da RBV em Penrose (1959), na obra The Theory of Growth of the Firm ("A teoria do crescimento da firma"). A teoria do crescimento - foco nominado do trabalho -, é desenvolvida como uma teoria de crescimento interno, de acordo com a qual a disponibilidade de recursos estimula a busca por oportunidades pelas quais se possam utilizá-los em prol da expansão das atividades da organização.

A expansão é decorrência da expectativa de se utilizarem os serviços provenientes dos recursos de modo mais rentável. É esse o incentivo que a organização tem para expandir. À medida que serviços de recursos são providos e não utilizados, deixa-se de se apropriar renda que poderia ser deles extraídas. Um programa de expansão implica, assim, um propósito e a sistematização necessária dos recursos para acompanhar esse propósito. 
Na década de 1980, Nelson e Winter (1982) em uma obra precursora - aprofundam a análise das rotinas administrativas da organização. As firmas difeririam em função das rotinas por meio das quais desempenham seus negócios - rotinas essas decorrentes dos conhecimentos humanos envolvidos nas operações. Rotinas são, pois, repositórios - e expressões - de conhecimento. O desenvolvimento de rotinas é, assim, a mais importante forma de estocagem de conhecimento, o lócus do conjunto de conhecimentos operacionais de uma organização.

$\mathrm{O}$ interesse no uso estratégico dos recursos foi reavivado por Wernerfelt (1984), com o objetivo de se entender o papel dos recursos na criação de vantagens competitivas. $\mathrm{O}$ autor propôs que o pioneirismo no uso de dado recurso tende a conferir à empresa pioneira uma vantagem de custo, porque, na medida em que vai adquirindo experiência, o recurso é utilizado de modo cada vez mais eficiente. O pioneirismo gera assim uma barreira de posição de recurso. Recursos atrativos são, portanto, aqueles cujo uso repetitivo conduz a decréscimos de custo, acarretando altos lucros; geralmente, trata-se de recursos ligados de modo semipermanente à organização, não disponíveis facilmente no mercado.

O autor também propõe que recursos exercem papel fundamental nas estratégias por meio das quais a vantagem competitiva é buscada pela diversificação. A combinação entre recursos atrativos correntes e novos recursos essenciais para se competir em outro mercado possibilita à organização ampliar suas vantagens de custo correntes e as barreiras delas decorrentes para novos mercados.

Continuando as análises de Wernerfelt, Barney (1991) organiza conceitos da teoria que se formava, a RBV. Recursos são definidos de modo amplo como todos os ativos, capacidades, processos organizacionais, atributos da firma, informação, conhecimento controlados pela firma, que a permitem conceber e implementar estratégias que aprimoram sua eficiência e sua efetividade. Podem ser classificados em físicos, humanos e organizacionais.

Os primeiros abrangem tecnologia física, planta e equipamento, localização geográfica e acesso a matérias-primas; os segundos, treinamento, experiência, julgamento, inteligência, relacionamentos e ideias de gestores e trabalhadores; os terceiros, a estrutura da firma, seus planejamentos formais e informais, sistemas de controle e coordenação, e também relacionamentos informais entre seus grupos, e entre a firma e aqueles que se acham no seu ambiente.

Sustentando-se sobre a dupla presunção de que recursos estratégicos não estão distribuídos equitativamente entre as firmas e de que essas diferenças são estáveis ao longo do tempo, o autor examina a ligação entre recursos e vantagem competitiva sustentável. Seu argumento orienta-se pela lógica de que não se consegue implementar uma estratégia que conduza à vantagem competitiva sustentável em um contexto de recursos homogêneos e perfeitamente móveis, do que decorre que apenas a heterogeneidade e a relativa imobilidade dos recursos podem servir como base ao desempenho superior sustentado.

Quatro indicativos empíricos básicos - valor, raridade, imitabilidade e substitutibilidade - são discutidos. Um recurso poderia ser fonte de vantagem competitiva sustentável quando valioso, raro, difícil de imitar e difícil de substituir. São essas as condições em função das quais um recurso pode ser considerado heterogêneo e não perfeitamente móvel, e, portanto, fonte de vantagem competitiva sustentável.

$\mathrm{O}$ valor de um recurso está relacionado ao seu potencial de proporcionar a exploração de oportunidades, ou a anulação de ameaças; a raridade, à sua escassez no mercado. Sendo um recurso valioso e raro, será provavelmente benéfico à organização. Mas apenas lhe proporcionarão vantagem sobre os concorrentes se forem também dificilmente imitáveis e dificilmente substituíveis.

A dificuldade de se imitar um recurso, segundo Barney (1991), advém de três elementos: condições históricas únicas, ambiguidade causal e complexidade social.

Cada organização é uma entidade social histórica, e sua habilidade de adquirir e explorar alguns recursos depende do ponto em que ela se encontra no tempo e no espaço. Quando recursos são criados ou adquiridos por condições históricas únicas, eles se tornam difíceis de imitar. O mesmo ocorre quando os concorrentes não conseguem entender como um recurso é utilizado para criar vantagem competitiva. Fala-se então em ambiguidade causal. Em outras palavras: não se compreende - ou se compreende apenas parcialmente - a ligação entre um recurso e a vantagem competitiva da organização.

A complexidade social, terceiro elemento, é o atributo de algo que, sendo complexo, se acha além da possibilidade de se gerenciar. Quando a vantagem competitiva está baseada em recursos constituintes de um fenômeno social complexo, a viabilidade de concorrentes imitarem esses recursos é significativamente restringida. Trata-se de recursos como relacionamentos interpessoais entre os gerentes, cultura da firma e reputação entre fornecedores e clientes.

Unicidade histórica, ambiguidade causal e complexidade social operam, assim, como elementos constritivos, impeditivos ou limitadores de movimentos miméticos.

A substitutibilidade, quarto indicativo, é ideia que emerge da relação entre recursos. Um recurso é insubstituível quando não há outro que seja em relação a ele um equivalente estratégico. A equivalência é medida de resultado. São equivalentes recursos que podem ser separadamente explorados, para implementar as mesmas estratégias. Coexistindo um 
substituto, há risco de que o recurso perca seu potencial de gerar vantagem.

O alcance da vantagem competitiva depende, pois, da implementação de uma estratégia de criação de valor que não possa ser ao mesmo tempo implementada por competidores atuais ou potenciais e cujos benefícios estratégicos não possam ser objeto de duplicação.

As implicações de mercados imperfeitos para recursos estrategicamente importantes haviam sido exploradas também por Dierickx e Cool (1989). Os autores referem-se ao processo de aquisição de recursos como "acumulação de estoque de ativos", motivo pelo qual se optou por preservar no texto a nomenclatura originariamente utilizada. Embasando sua tese em contraposições ao artigo de Barney (1986), os autores investigaram como as propriedades dinâmicas dos processos pelos quais as organizações constroem seus recursos afetam o potencial de renda desses recursos. O desenvolvimento interno de recursos é explicado por meio de uma metáfora.

A metáfora bath-tub comunica que "em qualquer momento, o estoque de água é indicado pelo nível de água existente na banheira; é o resultado cumulativo do fluxo prévio que jorra para dentro da banheira (pela torneira) e para fora dela (pelo ralo)" (Dierickx \& Cool, 1989, p. 1506). Em outros termos: a situação de ativos de uma organização em dado momento poderia ser entendida como a resultante da acumulação prévia de ativos obtida por meio da escolha de caminhos apropriados de fluxos ao longo de determinado período de tempo. Tal resultado é decorrente das escolhas estratégicas praticadas pela organização.

Os autores identificaram quatro propriedades dinâmicas de acumulação de estoque de ativos que previnem competidores do risco de terem seus recursos replicados: deseconomias de compressão de tempo; eficiências em massa de ativos; interconexão de ativos em estoque; ambiguidade causal.

Deseconomias de compressão de tempo elevam os custos de aquisição de certos tipos de recursos por concorrentes que tentam replicar rapidamente o estoque portado pela organização desses recursos. As deseconomias ocorrem porque, ao agilizar o uso desses recursos - o que se faz para antecipar seus efeitos desejados -, o competidor incorre em custos mais elevados que os que teria caso os desenvolvesse mais demoradamente. Dessa forma, a organização que já conta com estoque de recursos bem ajustados leva vantagem sobre aquelas que devem construí-los rapidamente (Dierickx \& Cool, 1989).

Eficiências em massa fazem com que o incremento do estoque de ativos torne-se mais eficiente na medida em que é aumentado. Dito de outra forma, o custo unitário de se adquirir mais de certos tipos de ativos decresce quando o estoque desses ativos é maior. As eficiências decorrem especialmente de dois motivos: já ter um determinado recurso torna mais fácil reconhecer, adquirir ou desenvolver mais dele próprio; tende a ser mais fácil integrar mais de um recurso quando a organização já o utiliza (Dierickx \& Cool, 1989).

A interconexão de ativos em estoque diminui a complicação de aumentar o estoque de certos ativos quando estoques de outros ativos já são importantes. Estoques amplos favorecem o desenvolvimento de novos estoques. Por fim, a ambiguidade causal ocorre quando o caminho que deve ser seguido para se desenvolver certo ativo não se mostra claro. Por consequência, torna-se difícil para os concorrentes identificar e replicar o processo de acumulação de recurso (Dierickx \& Cool, 1989; Barney, 1991).

Por essas razões, a implementação de uma estratégia pode requerer ativos que não são apropriáveis, que não estão disponíveis no mercado para aquisição. Não que só se produzam ativos não apropriáveis, mas são esses os ativos que têm maior potencial de gerar vantagem competitiva sustentável. Os apropriáveis podem ser livremente negociados no mercado. Por isso a estratégia demanda também ativos específicos da organização, desenvolvidos e acumulados internamente. Um estoque de ativos é tanto mais estratégico quanto menos negociáveis forem os ativos no mercado aberto. A posição de cada organização no mercado é determinada, assim, pelo nível de acúmulo de ativos importantes, consequência de fluxos prévios.

A perspectiva dos sistemas ampliou a discussão sobre as propriedades dos recursos no potencial de criação de vantagens competitivas (Sanchez \& Heene, 1996). Por essa perspectiva, reconhece-se a noção de base, ou sistema, de recursos e que a contribuição de um recurso na criação de valor depende de outros recursos que compõem o sistema no qual ele é utilizado. O potencial de um recurso pode, assim, ser ampliado ou contido em função da base que integra.

Dessa forma, alguns recursos comuns e disponíveis no mercado podem desempenhar um papel importante na consecução de vantagens competitivas. Esses recursos, a exemplo dos recursos de comunicação - como a internet - permitem à organização desempenhar atividades fundamentais como comunicar-se com agilidade. Embora não sejam raros, nem inimitáveis, e sejam movíveis entre as organizações (Barney, 1986), são cruciais ao apoio da criação de vantagens competitivas.

De acordo com as ideias apresentadas, é possível traçar algumas características de recursos que podem ser considerados estrategicamente relevantes, ou seja, podem contribuir para a criação e sustentação de estados de vantagem competitiva. $\mathrm{O}$ quadro abaixo sumariza essas características.

Recursos são analisados em função da estratégia que propiciam implementar. Como assinalam Mahoney e Pandian (1992) e Peteraf (1993), a posição de 
recursos é considerada já no conceito tradicional de estratégia - como em Andrews (1971) e Ansoff (1965). A organização seleciona suas estratégias para gerar renda baseada nas suas capacidades de recursos.

\section{PROCEDIMENTOS METODOLÓGICOS}

Foram consultadas, na base de dados Ebsco Business Source Complete -, nos periódicos
Administrative Science Quarterly, Academy of Management Review, Academy of Management Journal, Strategic Management Journal e Journal of Management todas as publicações em cujo abstract, ou author-supplied abstract, se encontra a expressão "resource based", e cujos textos completos se achavam disponíveis. 196 textos foram revisados.

\begin{tabular}{|c|c|}
\hline PERIÓDICO & VOLUME DE TEXTOS \\
\hline Academy of Management Journal & 28 \\
\hline Academy of Management Review & 25 \\
\hline Administrative Science Quarterly & 1 \\
\hline Journal of Management & 23 \\
\hline Strategic Management Journal & 119 \\
\hline Total & $\mathbf{1 9 6}$ \\
\hline
\end{tabular}

Figura 1 - Acervo revisado

Fonte: elaborada pelos autores.

Os periódicos foram selecionados por critério de notoriedade, por figurarem entre os principais veículos de comunicação da pesquisa científica produzida em Administração, e particularmente no campo da estratégia. Preservam, entre os periódicos genéricos ou seja, não limitados a campos específicos da área maior de business -, os mais elevados fatores de impacto (considerada a listagem de periódicos internacionais da área de business "2010 JCR Social Science Edition", divulgada pela Thomson Reuters). $\mathrm{Na}$ pesquisa bibliográfica promovida em estudos da RBV, os periódicos consultados são alvos recorrentes. Hoopes et al. (2003), por exemplo, referem-se à pesquisa bibliográfica realizada em quatro dos cinco periódicos selecionados: ASQ, AMJ, JOM e SMJ.

Ao vincular a busca da expressão ao abstract, ou author-supplied abstract, objetivou-se captar os materiais realmente pertinentes à RBV. É sabido que expressões referentes à RBV vêm sendo citadas em estudos de diversas áreas, muitos dos quais não utilizam a teoria como fundamento teórico. Restringindo o espaço de busca, restringiu-se também a probabilidade de se analisarem textos que não são propriamente "pertencentes" ao espaço teórico.

Pautando-se por critério de notoriedade, a expressão resource-based view (ou RBV) foi considerada em sentido amplo, designativa da teoria. Em geral, não distinguimos entre RBV e RBT (resource-based theory) - como, por exemplo, em Acedo, Barroso e Galan (2006), em que se classifica a RBV como corrente da RBT.

Utilizou-se a expressão "resource based", entre aspas, e sem hífen. Tencionou-se, com isso, englobar as variadas possibilidades de referência à RBV, como resource-based view, resource-based theory, resource- based perspective, resource-based model. O hífen foi suprimido entre as palavras componentes da expressão porque o procedimento de busca na base operada considera a expressão hifenizada mesmo quando não utilizado o hífen, mas não o faz em sentido inverso. Assim, utilizando, por exemplo, a expressão "resource based", captamos tanto textos em que aparece a expressão resource based quanto resource-based.

A revisão foi feita entre os meses de agosto e dezembro de 2011, e o conteúdo dos periódicos checado novamente - e fixado - em dezembro de 2011. A checagem ulterior - e fixação - do conteúdo foi praticada para readequar a base operacional a eventuais inclusões de textos ocorridas depois da primeira revisão.

\section{APRESENTAÇÃO E DISCUSSÃO DOS RESULTADOS}

\subsection{Características gerais da produção}

Os textos foram classificados conforme a natureza metodológica, o lócus de pesquisa e o tamanho da organização-objeto (ou das organizaçõesobjeto), a que se refere a pesquisa.

O método foi classificado em quantitativo, qualitativo, quantitativo-qualitativo (ou vice-versa), estudo teórico ou outro, conforme a ênfase metodológica do estudo.

Entre os textos classificados como estudos teóricos, estão artigos que não relatam pesquisas de campo, e revisões de livros (Book Reviews), introduções a edições especiais (special issues), referências a artigos premiados (prize-winning paper) e 
debates teóricos (dialogues) (estes, referenciados tal como apresentados na base de dados, unitariamente ou seja, não estão nominados individualmente, mas como dialogue, motivo pelo qual uma mesma referência pode indicar mais de um texto, já que, em geral, há compilados, sob uma mesma referência, mais de um debate. Há estudos teóricos nos quais aparecem exemplos ilustrativos, que, quando enfatizados no texto, e, sendo viável, geraram classificações quanto ao lócus e ao tamanho da(s) organização(ões). Os exemplos ilustrativos não enfatizados (quando a eles não se destina qualquer seção específica do texto) não foram classificados. Como outros, foram classificados textos não enquadrados nas demais modalidades, a exemplo de estudos experimentais.

O lócus refere-se ao espaço geográfico indicado no texto - ou como local de coleta (considerando-se também o local das fontes primárias de dados), ou como local a que se faz no texto referência vinculada ao conteúdo teórico sustentado. Organizações multinacionais (multinational enterprises ou MNEs) foram tratadas de acordo com a ênfase dada no texto a algum lócus específico - como, por exemplo, a sede da organização (headquarter). Nos textos que relatam estudos empíricos quantitativos ou qualitativos, é considerado o primeiro; nos demais, o segundo. Há textos em que não há indicação explícita do lócus. Foram classificados como prováveis os lócus dos quais não se tinha certeza - considerados como tais devido a evidências indicativas contidas nos textos. Na ausência de evidências percebidas, abstivemo-nos de classificar.

Para classificar o tamanho da organização, levaram-se em conta evidências variadas, buscadas especialmente na seção referente aos métodos utilizados (em methods ou em data and sample, por exemplo), ou, ainda, na seção destinada aos exemplos ilustrativos.

Foram buscados no texto - por meio do método automático de localização de palavras - termos como employee, sale, revenue, large, small, medium, SME, size, multinational. Procuraram-se, no entorno dessas palavras, indícios a respeito do tamanho da(s) organização(ões), como, por exemplo, o número de empregados, o faturamento, a indústria de atuação e a base da qual se extraíram os dados. A utilização de dados extraídos da base SPC (Standard \& Poor's Compustat), por exemplo, foi considerada - embora não isoladamente - um indício de referência a grandes organizações (ver Lin, Yang e Arya, 2009). Do mesmo modo, a utilização de dados extraídos da Fortune 500.

Nas tabelas abaixo, demonstram-se, de modo sintético, os indicativos obtidos.

Tabela 1 - Classificação dos textos analisados quanto ao método de pesquisa

\begin{tabular}{|c|c|c|c|c|c|c|c|c|c|c|c|c|}
\hline \multirow{2}{*}{$\begin{array}{c}\text { MÉTODO DE } \\
\text { PESQUISA }\end{array}$} & \multicolumn{2}{|r|}{ AMJ } & \multicolumn{2}{|c|}{ AMR } & \multicolumn{2}{|c|}{ ASQ } & \multicolumn{2}{|c|}{ SMJ } & \multicolumn{2}{|r|}{$\mathbf{J M}$} & \multicolumn{2}{|c|}{ TOTAL } \\
\hline & $\mathbf{F}$ & $\%$ & $\mathbf{F}$ & $\%$ & $\mathbf{F}$ & $\%$ & $\mathbf{F}$ & $\%$ & $\mathbf{F}$ & $\%$ & $\mathbf{F}$ & $\%$ \\
\hline Estudo teórico & 1 & $3,57 \%$ & 25 & $100 \%$ & 1 & $100 \%$ & 47 & $39,50 \%$ & 20 & $86,96 \%$ & 94 & $47,96 \%$ \\
\hline Estudo empírico & 27 & $96,43 \%$ & 0 & $0 \%$ & 0 & $0 \%$ & 70 & $58,82 \%$ & 3 & $13,04 \%$ & 100 & $51,02 \%$ \\
\hline Outro & 0 & $0 \%$ & 0 & $0 \%$ & 0 & $0 \%$ & 2 & $1,68 \%$ & 0 & $0 \%$ & 2 & $1,02 \%$ \\
\hline Total & 28 & $100 \%$ & 25 & $100 \%$ & 1 & $100 \%$ & 119 & $100 \%$ & 23 & $100 \%$ & 196 & $100 \%$ \\
\hline
\end{tabular}

Fonte: elaborada pelos autores.

Tabela 2 - Classificação dos estudos empíricos quanto ao método de pesquisa

\begin{tabular}{|l|c|c|c|c|c|c|c|c|c|c|c|c|}
\hline \multirow{2}{*}{$\begin{array}{c}\text { ESTUDOS } \\
\text { EMPÍRICOS }\end{array}$} & \multicolumn{2}{|c|}{ AMJ } & \multicolumn{2}{|c|}{ AMR } & \multicolumn{2}{|c|}{ ASQ } & \multicolumn{2}{|c|}{ SMJ } & \multicolumn{2}{c|}{ JM } & \multicolumn{2}{c|}{ TOTAL } \\
\cline { 2 - 14 } & $\mathbf{F}$ & $\mathbf{\%}$ & $\mathbf{F}$ & $\mathbf{\%}$ & $\mathbf{F}$ & $\mathbf{\%}$ & $\mathbf{F}$ & $\mathbf{\%}$ & $\mathbf{F}$ & $\mathbf{\%}$ & F & \% \\
\hline Quanti & 26 & $96,30 \%$ & 0 & $0 \%$ & 0 & $0 \%$ & 61 & $87,14 \%$ & 2 & $66,67 \%$ & 89 & $89 \%$ \\
\hline Quali & 0 & $0 \%$ & 0 & $0 \%$ & 0 & $0 \%$ & 6 & $8,57 \%$ & 0 & $0 \%$ & 6 & $6 \%$ \\
\hline Quali/Quanti & 1 & $4 \%$ & 0 & $0 \%$ & 0 & $0 \%$ & 3 & $4,29 \%$ & 1 & $33,33 \%$ & 5 & $5 \%$ \\
\hline Total & 27 & $100 \%$ & 0 & $0 \%$ & 0 & $0 \%$ & 70 & $100 \%$ & 3 & $100 \%$ & 100 & $100 \%$ \\
\hline
\end{tabular}

Fonte: elaborada pelos autores. 
A Produção Internacional em RBV: Características Gerais e Tratativa dos Recursos na Pesquisa em Organizações de Menor Porte

Tabela 3 - Classificação dos estudos que contêm indicação de lócus

\begin{tabular}{|l|c|c|c|c|c|c|c|c|c|c|c|c|}
\hline \multirow{2}{*}{ LÓCUS } & \multicolumn{2}{|c|}{ AMJ } & \multicolumn{2}{|c|}{ AMR } & \multicolumn{2}{c|}{ ASQ } & \multicolumn{2}{|c|}{ SMJ } & \multicolumn{2}{c|}{ JM } & \multicolumn{2}{c|}{ TOTAL } \\
\cline { 2 - 14 } & $\mathbf{F}$ & $\boldsymbol{\%}$ & $\mathbf{F}$ & $\boldsymbol{\%}$ & $\mathbf{F}$ & $\boldsymbol{\%}$ & $\mathbf{F}$ & $\boldsymbol{\%}$ & $\mathbf{F}$ & $\boldsymbol{\%}$ & F & \% \\
\hline Inclusive EUA $^{\mathrm{a}}$ & 21 & $75 \%$ & 3 & $75 \%$ & 1 & $100 \%$ & 56 & $76,71 \%$ & 2 & $100 \%$ & 83 & $76,85 \%$ \\
\hline Sem EUA & 7 & $25 \%$ & 1 & $25 \%$ & 0 & $0 \%$ & 17 & $23,29 \%$ & 0 & $0 \%$ & 25 & $23,15 \%$ \\
\hline Total & 28 & $100 \%$ & 4 & $100 \%$ & 1 & $100 \%$ & 73 & $100 \%$ & 2 & $100 \%$ & 108 & $100 \%$ \\
\hline
\end{tabular}

Fonte: elaborada pelos autores.

a O grupo "inclusive EUA" considera também os estudos qualificados por "provavelmente".

Tabela 4 - Classificação dos estudos que contêm indicação de tamanho

\begin{tabular}{|c|c|c|c|c|c|c|c|c|c|c|c|c|}
\hline \multirow{2}{*}{$\begin{array}{l}\text { TAMANHO DAS } \\
\text { ORGANIZAÇÕES }\end{array}$} & \multicolumn{2}{|c|}{ AMJ } & \multicolumn{2}{|c|}{ AMR } & \multicolumn{2}{|c|}{ ASQ } & \multicolumn{2}{|c|}{ SMJ } & \multicolumn{2}{|r|}{ JM } & \multicolumn{2}{|c|}{ TOTAL } \\
\hline & $\mathbf{F}$ & $\%$ & $\mathbf{F}$ & $\%$ & $\mathbf{F}$ & $\%$ & $\mathbf{F}$ & $\%$ & $\mathbf{F}$ & $\%$ & $\mathbf{F}$ & $\%$ \\
\hline Pequena(s), média(s) & 2 & $7 \%$ & 0 & $0 \%$ & 0 & $0 \%$ & 5 & $6,85 \%$ & 1 & $33,33 \%$ & 8 & $7,48 \%$ \\
\hline Grande(s) & 21 & $78 \%$ & 4 & $100 \%$ & 0 & $0 \%$ & 56 & $76,71 \%$ & 2 & $66,67 \%$ & 83 & $77,57 \%$ \\
\hline Variado & 4 & $15 \%$ & 0 & $0 \%$ & 0 & $0 \%$ & 12 & $16,44 \%$ & 0 & $0 \%$ & 16 & $14,95 \%$ \\
\hline Total & 27 & $100 \%$ & 4 & $100 \%$ & 0 & $0 \%$ & 73 & $100 \%$ & 3 & $100 \%$ & 107 & $100 \%$ \\
\hline
\end{tabular}

Fonte: elaborada pelos autores.

${ }^{a}$ O grupo "pequena(s), média(s)" constitui-se pelas classificações "pequena(s)", "média(s)", "pequena(s) e média(s)" e "provavelmente" quaisquer delas. O grupo "grande(s)" constitui-se pelas classificações "grande(s)" e "provavelmente grande(s)". O grupo "variado" constituições pelas classificações "variável” e "provavelmente variado".

A extensa variação temática dos textos publicados é característica marcante da publicação: a RBV é uma teoria que se permite ao trânsito excursionista de diversas teorias, de distintos campos. Há entre os textos analisados, entre outros, estudos relacionados às áreas de recursos humanos, negócios internacionais, finanças, privatização, fusões e aquisições, gestão ambiental, alianças estratégicas, empreendedorismo, teoria dos jogos. São exemplos, respectivamente: Ployhart, Van Iddekinge e Mackenzie Jr. (2011), Yaping (2003), Vincente-Lorente (2001), Makhija (2003), Capron (1999), Aragón-Correa e Sharma (2003), Dyer e Singh (1998), Wiklund e Shepherd (2003), Knez e Camerer (1994). São, enfim, diversas as teorias com as quais a RBV é relacionadao que parece torná-la profícua, e dotada de identidade um tanto alargada.

Os periódicos - pôde-se notar - têm perfis bastante distintos. No Administrative Science Quarterly, apenas um texto foi filtrado, enquanto no Strategic Management Journal foram filtrados cento e dezenove. No Academy of Management Review, entre os vinte e cinco textos filtrados, não há estudo empírico, enquanto no Academy of Management Journal, dos vinte e oito textos filtrados, apenas um é estudo teórico.

Grande parte dos estudos ainda é de natureza teórica $-47,96 \%$. Entre os estudos empíricos, o método predominante é o quantitativo - $89 \%$. Somando-se aos estudos que se utilizam de método "quanti-quali", esse percentual sobe para 94\%. Apenas 6\% dos estudos empíricos são puramente qualitativos, do que se pode concluir que a abordagem na pesquisa em RBV é tendenciosamente quantitativa $-\mathrm{o}$ que, por consequência, talvez em via oposta, influencia a tipologia da problemática pesquisada.

Entre os textos que se referem a algum lócus geográfico, a maior parte inclui referência a organizações americanas $-76,85 \%$. Entre os textos que se referem a organizações como fontes de dados, a maior parte inclui referência a grandes organizações somente - 77,57\% (Incluindo-se a classificação "provavelmente grande"). Assim se tem estruturado a pesquisa em RBV no conjunto analisado.

\subsection{A pesquisa em organizações de menor porte: características temáticas e tratativa de recursos}

Dos textos que contêm indicação de tamanho, oito referem-se a pequenas ou médias organizações. Abordam temas variados, e variados são também os modos como se lida com a teoria. No quadro abaixo, apresenta-se resumidamente, em referência a cada texto, a diretriz de pesquisa norteadora do estudo e a tratativa sob a qual se consideram os recursos. 


\begin{tabular}{|c|c|c|}
\hline TEXTO & DIRETRIZ DE PESQUISA & TRATATIVA \\
\hline $\begin{array}{l}\text { Mesquita, L. F. \& Lazzarini, S. G. (2008) } \\
\text { Horizontal and vertical relationships in } \\
\text { developing economies: implications for SME's } \\
\text { access to global markets. Academy of } \\
\text { Management Journal, 51(2): 359-380. }\end{array}$ & $\begin{array}{l}\text { Integram-se três teorias - RBV, TCE, e teoria } \\
\text { institucional - para modelar como a } \\
\text { colaboração entre PMEs em ambientes } \\
\text { formados por fraca infraestrutura e fracas } \\
\text { instituições ajudam-nas a atingir maiores } \\
\text { eficiências coletivas e acesso a mercados } \\
\text { globais. }\end{array}$ & $\begin{array}{l}\text { Recursos e vantagens como } \\
\text { provenientes das redes locais de } \\
\text { negócio. }\end{array}$ \\
\hline $\begin{array}{l}\text { Katila, R. \& Shane, S. (2005) When does lack } \\
\text { of resources make new firms innovative? } \\
\text { Academy of Management Journal, 48(5): 814- } \\
829 .\end{array}$ & $\begin{array}{l}\text { Estende-se a RBV para explicar a inovação } \\
\text { em novos empreendimentos que, carecedores } \\
\text { de recursos, ainda têm que desenvolvê-los. }\end{array}$ & $\begin{array}{l}\text { Pesquisa direcionada à falta de } \\
\text { recursos. Questiona-se quando - isto é, } \\
\text { sob que condições ambientais - é a } \\
\text { falta de recursos fator útil ou inibidor } \\
\text { da inovação. }\end{array}$ \\
\hline $\begin{array}{l}\text { Li, H. \& Zhang, Y. (2007). The role of } \\
\text { managers' political networking and functional } \\
\text { experience in new venture performance: } \\
\text { Evidence from China's transition economy. } \\
\text { Strategic Management Journal, 28(8): 791-804. }\end{array}$ & $\begin{array}{l}\text { Utilizam-se a RBV e a TCE para examinar } \\
\text { como dois tipos de recursos - relações } \\
\text { políticas e experiência funcional dos gestores } \\
\text { - estão relacionados ao desempenho de novos } \\
\text { negócios em economias em transição. }\end{array}$ & $\begin{array}{l}\text { Recursos como relacionamento } \\
\text { político e experiência funcional - } \\
\text { ambos referentes aos gestores. }\end{array}$ \\
\hline $\begin{array}{l}\text { Wiklund, J. \& Shepherd, D. A. (2003). } \\
\text { Knowledge-based resources, entrepreneurial } \\
\text { orientation, and the performance of small and } \\
\text { medium-sized businesses. Strategic } \\
\text { Management Journal, 24(13): 1307-1314. }\end{array}$ & $\begin{array}{l}\text { Sugere-se que recursos baseados no } \\
\text { conhecimento (aplicáveis à descoberta e } \\
\text { exploração de oportunidades) relacionam-se } \\
\text { positivamente ao desempenho } \\
\text { organizacional, e que a orientação } \\
\text { empreendedora reforça essa relação. }\end{array}$ & $\begin{array}{l}\text { Recursos como provenientes do } \\
\text { conhecimento (considerados } \\
\text { conhecimento do mercado e } \\
\text { conhecimento tecnológico). }\end{array}$ \\
\hline $\begin{array}{l}\text { Knott, A. M. (2003). The organizational } \\
\text { routines factor market paradox. Strategic } \\
\text { Management Journal, 24(10): 929-943. }\end{array}$ & $\begin{array}{l}\text { Considerando-se proposição da RBV de que } \\
\text { rotinas organizacionais superiores podem ser } \\
\text { fonte de valor apenas e quando houver } \\
\text { mecanismos de isolamento que previnam sua } \\
\text { difusão pela indústria, levanta-se um } \\
\text { paradoxo a se resolver: a existência de } \\
\text { rotinas explícitas, porém valiosas, evidente } \\
\text { no sistema de franquias. }\end{array}$ & $\begin{array}{l}\text { Recursos como rotinas superiores ( } a \\
\text { routines view of franchise). }\end{array}$ \\
\hline $\begin{array}{l}\text { Spanos, Y. E. \& Lioukas, S. (2001). An } \\
\text { examination into the causal logic of rent } \\
\text { generation: contrasting Porter's competitive } \\
\text { strategy framework and the resource-based } \\
\text { perspective. Strategic Management Journal, } \\
\text { 22(10): 907-934. }\end{array}$ & $\begin{array}{l}\text { Compõem-se premissas de duas correntes da } \\
\text { estratégia - Porter's competitive strategy e } \\
\text { RBV - para se criar um modelo explicativo } \\
\text { do desempenho organizacional. }\end{array}$ & $\begin{array}{l}\text { Recursos genericamente considerados } \\
\text { como capacidades organizacionais, de } \\
\text { mercado e técnicas. }\end{array}$ \\
\hline $\begin{array}{l}\text { Lorenzoni, G. \& Lipparini, A. (1999). The } \\
\text { leveraging of interfirm relationships as a } \\
\text { distinctive organizational capability: a } \\
\text { longitudinal study. Strategic Management } \\
\text { Journal, 20(4): 317-338. }\end{array}$ & $\begin{array}{l}\text { Alimenta-se a discussão sobre alianças } \\
\text { cooperativas e os aspectos dinâmicos das } \\
\text { relações interfirmas. Argumenta-se que a } \\
\text { capacidade de interagir com outras } \\
\text { companhias acelera o acesso ao } \\
\text { conhecimento, e sua transferência. }\end{array}$ & $\begin{array}{l}\text { Recurso como capacidade de } \\
\text { relacionamento (relational capability). }\end{array}$ \\
\hline $\begin{array}{l}\text { Mosakowski, E. (1993). A Resource-Based } \\
\text { Perspective on the Dynamic Strategy- } \\
\text { Performance Relationship: An Empirical } \\
\text { Examination of the Focus and Differentiation } \\
\text { Strategies in Entrepreneurial Firms. Journal of } \\
\text { Management, 19(4), 819-839. }\end{array}$ & $\begin{array}{l}\text { Aplica-se a RBV à questão sobre como as } \\
\text { estratégias de foco ou diferenciação afetam o } \\
\text { desempenho econômico de firmas } \\
\text { empreendedoras. }\end{array}$ & $\begin{array}{l}\text { Recursos genericamente considerados } \\
\text { - associados à determinada escolha } \\
\text { estratégica (foco ou diferenciação). }\end{array}$ \\
\hline
\end{tabular}

Figura 2 - Diretriz de pesquisa e tratativa conferida aos recursos em estudos estrangeiros que se referem a pequenas ou médias organizações

Fonte: elaborada pelos autores. 
Dos oito artigos, seis apresentam pesquisas quantitativas, um, pesquisa quantitativa-qualitativa, e um, pesquisa qualitativa. Quase todas as pesquisas partem de tipos específicos de recursos, definidos previamente na literatura, para testá-los nas organizações-objeto da pesquisa.

Os artigos de Mosakowsky (1993) e Spanos e Lioukas (2001) abordam os recursos sob tratativa genérica. Aquele enfatiza capacidades, em três classes - organizacionais, técnicas e de mercado; este os considera em associação a escolhas estratégicas genéricas, "porterianas" - foco ou diferenciação. Os demais artigos abordam os recursos de modo mais especificado: como provenientes de redes sociais; como relacionamento político e experiência funcional dos gestores; como provenientes do conhecimento, de mercado e tecnológico; como rotinas superiores; e como capacidade de relacionamento.

Sob formas variadas, o relacionamento interorganizacional é apontado correntemente na pesquisa como um recurso fundamental à prática estratégica de PME's (Pihkala, Varamaki \& Vesalainen, 1999; Meyskens, Carsrud \& Cardozo, 2010; Saes, 2010) - como se nota, no subconjunto analisado, em Mesquita e Lazzarini (2008) e em Lorenzoni e Lipparini (1999). Não apenas, mas destacadamente, na ausência de estruturas de que se dotam o mercado ou organizações de maior porte, a capacidade de relacionamento com outras firmas é tida por essencial.

Os relacionamentos possibilitam o desenvolvimento conjunto, a viabilização de práticas estratégicas. Laços cooperativos podem operar como estimuladores de conhecimento e inovação (Lorenzoni \& Lipparini, 1999); relacionamentos são utilizados para se conquistarem eficiências coletivas (Mesquita \& Lazzarini, 2008), criar e fortalecer marca (Saes, 2010) ou mesmo, e em importante medida, para se adquirirem recursos (Meyskens et al., 2010).

Destaque também se tem conferido a recursos relacionados à aprendizagem, ao conhecimento e às rotinas organizacionais. O debate ocorre sob o respaldo de trabalhos notórios da teoria - como Nelson e Winter (1982) e Grant (1996). Fundamentalmente, atribui-se à qualidade do tácito - característica dos referidos recursos - um manto de estima de grande valia, que torna os recursos que distingue os da firma mais estrategicamente significantes.

O conhecimento - representando o intangível marca o modo como se utilizam os recursos - sendo, por si, também um recurso (Wiklund \& Shepherd, 2003); rotinas valiosas, protegidas por mecanismos de isolamento - ainda mesmo que em função de falhas de pretensos imitadores - podem ser ativos geradores de valor (Knott, 2003).

O artigo de Katila e Shane (2005) destaca-se dos demais. A pesquisa relaciona a falta de recursos - fator tipicamente constatado em organizações iniciantes, e de menor porte - e o nível de inovação. Questiona-se quando a carência de recursos serve como fato útil ou inibidor da inovação. Haveria condições ambientais nas quais a falta de recursos é fator útil à inovação; um fator a priori negativo - com o qual usualmente se deparam novas e também pequenas organizações - poderia, por vezes, assim, representar alguma utilidade à inovação. Temática próxima, a dificuldade financeira é abordada, no campo do empreendedorismo, em Madrid-Guijarro, GarcíaPérez-de-Lema e Van Auken (2011).

As pesquisas apresentadas nos artigos não têm tido como foco a identificação de recursos específicos. Embasam-se em recursos específicos, previamente abordados na literatura - esta, predominantemente voltada para grandes organizações -, e, sob o referencial correspondente, são desenhados os métodos de investigação direcionados à realidade das pequenas e médias organizações que lhes servem de objeto.

Com exceção da pesquisa de Katila e Shane (2005), que se presta a associar a falta de recursos ao grau de inovação organizacional, procedem-se as demais por conta, por assim dizer, da experimentação de recursos, na ambiência de organizações de menor porte. A predominância de métodos quantitativos indica, aliás, que as construções metodológicas das pesquisas contidas nos artigos orientam-se primordialmente por variáveis, cuja concepção não se funda na realidade observada tanto quanto na literatura que a precede. Esse indicativo é ratificado com a leitura dos textos.

\section{CONSIDERAÇÕES FINAIS}

Este artigo relata parte de um estudo cujo objetivo foi gerar aprofundamentos no estado da arte da RBV. Expõe-se aqui a seção destinada à caracterização geral do campo e à tratativa conferida aos recursos na pesquisa em RBV que lida com organizações de menor porte. A fim de se realizar o propósito traçado, estipularam-se objetivos intermediários pelos quais se orientaram os esforços de pesquisa.

Com a finalidade de conhecer os textos que integram os acervos de veículos importantes da área, procedeu-se à busca, na base da Ebsco, dos textos que se apoiam de alguma forma nos postulados da RBV, cumprindo-se, dessa forma, o primeiro objetivo intermediário. A fim de viabilizar a caracterização da produção compilada, constituiu-se um conjunto de indicativos a partir dos quais foram os textos categorizados, conforme proposta do segundo objetivo intermediário. O desenvolvimento das categorias analíticas utilizadas pautou-se nos seguintes critérios: retratar qualidades epistemológicas elementares da 
pesquisa - se teóricas, empíricas ou de outro tipo e, sendo empíricas, se procedidas por métodos quantitativos, qualitativos ou mistos; e averiguar proposições veiculadas na literatura - quanto à predominância, no que tange ao objeto de pesquisa, do lócus americano e da organização de grande porte.

As análises procederam-se, assim, em parte como meio de compreensão geral da produção e em parte como reflexo a demandas propositivas preexistentes na literatura. Na seção de apresentação e discussão dos resultados, salientaram-se algumas discussões.

Vale registrar que, impregnando-se das constatações objetivas - emergidas da necessária relação havida entre teoria e objeto -, as lógicas formatadoras da teoria amoldam-se aos fenômenos que, objetos das pesquisas empíricas, servem-na como nutriz. Assim, não apenas o campo da estratégia em geral, como também a RBV, têm-se constituído por construtos modelados principalmente para a finalidade de análise da grande organização. Na RBV, como demonstrado, tal ocorrência é evidente. A constatação de Barney, Wright e Ketchen (2001), que apontava para a possibilidade de extensão da teoria para a consideração de empresas menores, persiste atual, transcorrida mais de uma década.

O mesmo se pode afirmar da observação de Whittington et al. (2002) sobre a preponderância da lócus americano como espaço do qual são extraídas as organizações objetos de estudo. Nos acervos analisados, mais de $75 \%$ dos estudos que contêm indicação de lócus referem-se a organizações americanas. O possível viés havido por conta da origem das instituições às quais os periódicos são vinculados não serve a desfazer a notabilidade tampouco as consequências práticas decorrentes - do fato de que as principais publicações reportam pesquisas empíricas realizadas em organizações americanas.

A categorização quanto ao porte permitiu abstrair dos textos compilados aqueles que relatam pesquisas realizadas em organizações de menor porte, cumprindo-se, assim, o terceiro objetivo intermediário. Constatou-se que apenas pouco mais de $7 \%$ dos estudos que contêm indicação de tamanho referem-se a pequenas ou médias organizações. A análise de cada um dos artigos componentes desse subconjunto proporcionou que se identificasse e se categorizasse as tratativas neles conferidas aos recursos, satisfazendose, assim, o quarto objetivo intermediário estipulado.

De modo geral, nota-se que, mesmo quando se opta por pesquisar organizações de menor porte, a tratativa conferida aos recursos ainda se dota do viés, próprio do campo, da pesquisa que toma por objeto grandes organizações. Tal consideração abre espaço para, talvez por meio do uso de métodos qualitativos, por meio dos quais se possa gerar investigações mais próximas do objeto e menos atreladas às constatações prévias registradas na literatura, desenvolverem-se pesquisas que tenham por propósito a compreensão dos recursos no âmbito particular da organização de menor porte. Em outras palavras, não se sequenciarem, como muitas vezes praticado em estudos nos quais se aplica a RBV à realidade de PMEs, aplicações metodológicas tradicionais da teoria (em extensa medida, representadas por métodos investigatórios quantitativos, que, no conjunto analisado, somam $89 \%$ das aplicações praticadas nos estudos empíricos, e, considerando-se também a conjugação "Quali/Quanti”, 94\%), mas permitir-se à percepção intrinsecamente contextualizada dos recursos sobre os quais têm operado organizações de menor porte. Entende-se que pode haver também aí um relevante domínio para pesquisas futuras - em outras palavras,

O método utilizado - explicitado na seção de procedimentos metodológicos e, suplementarmente, antes da apresentação dos dados - também pode ser útil como modelo para a condução de outros de levantamento. Além de conferir transparência ao relato dos percursos conducentes da pesquisa - o que, em última análise, torna claro e passível de compartilhamento o conceito nominal pelo qual se refere à amostra -, permite ao pesquisador exercer um trabalho relativamente organizado, o que pode ser fundamental quando se lida com acervos demasiado extensos. Os repositórios digitais, ao mesmo que tempo que proporcionam grandes avanços à pesquisa bibliográfica, também impõem o desafio de se saber lidar com os pormenores de métodos automáticos de busca e com a categorização do grande volume de materiais a que permitem acesso.

Acompanhando a sugestão pela impressão de mais contexto na pesquisa, para se estender a teoria para a considerações de organizações de menor porte, inseridas em contextos regionais menos amplos - o que se alinha com a agenda proposta por Whittington et al. (2002) -, sugere-se a averiguação da possibilidade de se exercerem algumas alterações estruturais na seleção dos destinos da aplicação da RBV: i) de grandes companhias para organizações de menor porte; ii) de organizações líderes de mercado ou em busca da liderança para boas organizações; iii) do lócus centrado na faixa de liderança para lócus esparsos, em nível regional; iv) da busca pela explicação dos melhores desempenhos para a busca pela explicação de bons desempenhos, satisfatórios.

Entre as limitações da pesquisa realizada, saliente-se a referente à composição do conjunto de acervos analisado. Se, por um lado, representa a pesquisa mais impactante sobre o campo teórico dando em princípio a conhecer as experiências mais difundidas pelo campo -, por outro, exclui publicações de efeitos menos amplos, nas quais talvez se encontrassem os lapsos mencionados como resultados do estudo realizado. Essa limitação sugere que se estenda a pesquisa a composições distintas da 
selecionada, proporcionando que se comparem os resultados.

\section{REFERÊNCIAS}

Acedo, F. J., Barroso, C. \& Galan, J. L. (2006). The resource-based theory: dissemination and main trends. Strategic Management Journal, 27(7): 621636.

Andrews, K. R. (1971). The Concept of Corporate Strategy. Homewood, Ill: Dow Jones-Irwin.

Ansoff, H. I. (1965). Corporate Strategy. New York: McGraw-Hill.

Aragón-Correa, J. A. \& Sharma, S. (2003). A contingent resource-based view of proactive corporate environmental strategy. Academy of Management Review, 28(1): 71-88.

Barney, J. (1986). Strategic Factor Markets: expectations, luck, and business strategy. Management Science, 32(10): 1231-1241.

Barney, J. (1991). Firm Resources and Sustained Competitive Advantage. Journal of Management, 17(1): 99-120.

Barney, J., Wright, M. \& Ketchen Jr., D. J. (2001) The resource-based view of the firm: Ten years after 1991. Journal of Management, 27(6), 625-641.

Capron, L. (1999). The long-term performance of horizontal acquisitions. Strategic Management Journal, 20(11): 987-1018.

Dierickx, I. \& Cool, K. (1989). Asset stock accumulation and sustainability of competitive advantage. Management Science, 35(12): 15041511.

Dyer, J. H. \& Singh, H. (1998). The relational view: Cooperative strategy and sources of interorganizational competitive advantage. Academy of Management Review, 23(4), 660-680.

Grant, R. M. (1996). Toward a knowledge-based theory of the firm. Strategic Management Journal, 17: 109-122.

Hoopes, D. G., Madsen, T. L. \& Walker, G. (2003). Guest Editors' introduction to the special issue: Why is there a resource-based view? Toward a theory of competitive heterogeneity. Strategic Management Journal, 24(10): 889-902.
Katila, R. \& Shane, S. (2005) When does lack of resources make new firms innovative? Academy of Management Journal, 48(5): 814-829.

Knez, M. \& Camerer, C. (1994). Creating expectational assets in the laboratory: coordination in 'weakest-link' games. Strategic Management Journal, 15: 101-119.

Knott, A. M. (2003). The organizational routines factor market paradox. Strategic Management Journal, 24(10): 929-943.

Li, H. \& Zhang, Y. (2007). The role of managers' political networking and functional experience in new venture performance: Evidence from China's transition economy. Strategic Management Journal, 28(8): 791-804.

Lin, Z. J., Yang, H. \& Arya, B. (2009). Alliance partners and firm performance: resource complementarity and status association. Strategic Management Journal, 30(9): 921-940.

Lorenzoni, G. \& Lipparini, A. (1999). The leveraging of interfirm relationships as a distinctive organizational capability: a longitudinal study. Strategic Management Journal, 20(4): 317-338.

Madrid-Guijarro, A., García-Pérez-De-Lema, D. \& Van Auken, H. (2011). An analysis of non-financial factors associated with financial distress. Entrepreneurship \& Regional Development, 23(3/4): 159-186.

Mahoney, J. T. \& Pandian, J. R. (1992). The ResourceBased View Within Conversation of Strategic Management. Strategic Management Journal, 13(5): 363-380.

Makhija, M. (2003). Comparing the resource-based and market-based views of the firm: empirical evidence from Czech privatization. Strategic Management Journal, 24(5): 433-451.

Mesquita, L. F. \& Lazzarini, S. G. (2008) Horizontal and vertical relationships in developing economies: implications for SME's access to global markets. Academy of Management Journal, 51(2): 359-380.

Meyer, G. D., Neck, H. M. \& Meeks, M. D. (2002). The Entrepreneurship-Strategic Management Interface. In Hitt, M. A., Ireland, R. D., Camp, S. \& Sexton, D. L. (ed.) Strategic Entrepreneurship: creating a new mindset, pp. 19-44, Oxford: Blackwell Publishers.

Meyskens, M., Carsrud, A. L. \& Cardozo, R. N. (2010). The symbiosis of entities in the social 
engagement network: The role of social ventures. Entrepreneurship \& Regional Development, 22(5): 425-455.

Mosakowski, E. (1993). A Resource-Based Perspective on the Dynamic Strategy-Performance Relationship: An Empirical Examination of the Focus and Differentiation Strategies in Entrepreneurial Firms. Journal of Management, 19(4), 819-839.

Nelson, R. R. \& Winter, S. G. (1982). An Evolutionary Theory of Economic Change. Cambridge (MA): Harvard University Press.

Newbert, S. L. (2007). Empirical research on the resource-based view of the firm: an assessment and suggestions for future research. Strategic Management Journal, 28(2): 121-146.

Penrose, E. T. The Theory of Growth of the Firm. London: Basil Blackwell, 1959.

Penrose, E. T. (1997). The Theory of the Growth of the Firm. In Foss, N., J. (ed.) Resources, firms and strategy: a reader in the resource-based perspective, pp. 27-39, New York: Oxford University Press.

Peteraf, M. A. (1993). The Cornerstones of Competitive Advantage: A Resource-Based View. Strategic Management Journal, 14(3): 179-191.

Pihkala, T., Varamaki, E. \& Vesalainen, J. (1999). Virtual organization and the SMEs: a review and model development. Entrepreneurship \& Regional Development, 11(4): 335-349.

Ployhart, R. E., Van Iddekinge, C. H. \& Mackenzie Jr., W. I. (2011). Acquiring and developing human capital in service contexts: the interconnectedness of human capital resources. Academy of Management Journal, 54(2): 353-368.

Porter, M. E. (1980). Competitive Strategy: techniques for analyzing industries and competitors. New York: The Free Press.
Saes, M. S. M. (2010). Rent appropriation among rural entrepreneurs: three experiences in coffee production in Brazil. Revista de Administração da USP, 45(4): 313-327.

Sanchez, R. \& Heene, A. (1996). A Systems View of the Firm in Competence-Based Competition. In Sanchez, R., Heene, A. \& Thomas, H. (ed.) Dynamics of Competence-Based Competition: Theory and Practice in the New Strategic Management, pp. 39-62, Oxford: Elsevier Pergamon.

Spanos, Y. E. \& Lioukas, S. (2001). An examination into the causal logic of rent generation: contrasting Porter's competitive strategy framework and the resource-based perspective. Strategic Management Journal, 22(10): 907-934.

Stieglitz, N. \& Heine, K. (2007). Innovations and the role of complementarities in a strategic theory of the firm. Strategic Management Journal, 28(1): 115.

Vincente-Lorente, J. D. (2001). Specificity and opacity as resource-based determinants of capital structure: evidence for Spanish manufacturing firms. Strategic Management Journal, 22(2): 157-177.

Wernerfelt, B. (1984). A resource-based view of the firm. Strategic Management Journal, 5(2): 171180.

Wiklund, J. \& Shepherd, D. A. (2003). Knowledgebased resources, entrepreneurial orientation, and the performance of small and medium-sized businesses. Strategic Management Journal, 24(13): 1307-1314.

Whittington, R., Pettigrew, A. \& Thomaz, H. (2002). Conclusion: doing more in strategy research. In Pettigrew, A. \& Thomaz, H., Whittington, R. (ed.) Handbook of Strategy and Management, pp. 475488, London: Sage Publications.

Yaping, G. (2003). Subsidiary staffing in multinational enterprises: agency, resources, and performance. Academy of Management Journal, 46(6): 728-739. 